Vasopressor boluses (Phenylephrine $50 \mathrm{mcg} / \mathrm{ml}$ ) were administered if the MAP dropped by $10 \%$ of the initial value.

The number of bolused episodes was recorded.

All patients received an IV balanced crystalloid $(10 \mathrm{ml} / \mathrm{kg})$

at $\mathrm{SAB}$ insertion, followed by an infusion.

Pain scores (VAS) were documented perioperatively.

Results Conclusions

1. Slowly titrated, low-dose SAB in elderly patients with mildmoderate, asymptotic AS did not develop precipitous hypotension.

2. MAP was relatively easily maintained with boluses of Phenylephrine $(50 \mathrm{mcg} / \mathrm{ml})$.

3. US-guided SIFI FICB is an effective analgesic adjunct.

\section{CONTINUOUS SPINAL ANESTHESIA FOR OPEN CHOLECYSTECTOMY: CUSTOMIZED ANAESTHETIC MANAGEMENT IN A CHALLENGING PATIENT}

C Lopes*, D Zuzarte, M Correia. Centro Hospitalar de Lisboa Ocidental, Lisboa, Portugal

\subsection{6/rapm-2021-ESRA.55}

Background and Aims Continuous spinal anesthesia (CSA) provides fast onset titratable neuroaxial block. In the context of upper abdominal surgery on patients with acute respiratory disease offers minimization of respiratory complications and ensures adequate anaesthetic conditions.

We aim to present a CSA for open cholecystectomy in a patient with multiple neurological deficits and acute pneumonia.

Methods 60 years-old woman, $40 \mathrm{~kg}, 152 \mathrm{~cm}$. Past history of rickets having severe skeletal deformation; hypertension; status post cerebellar tumor resection with multiple sequelae: loss of visual and auditory acuity, dysphagia, dysphonia, respiratory insufficiency and ventriculoperitoneal shunt. Admitted for urgent open cholecystectomy having alongside acute pneumonia with pleural effusion. Laboratory tests and current medication posed no contraindication to spinal anesthesia.

Spinal catheter 20G was introduced through $18 \mathrm{G}$ tuohy needle at L3-L4 level. On supine position the adequate block level was achieved using bed tilting and sequential doses of hyperbaric bupivacaine (total of $10 \mathrm{mg}$ ) and sufentanil (total of $10 \mathrm{mcg}$ ). During the $1 \mathrm{~h}$ procedure there was no need for sedation. The spinal catheter was removed on the operatory room (OR) after a morphine $100 \mathrm{mcg}$ administration. Neither respiratory nor regional anesthesia complications were reported on the postoperative period.

Results Although a CSA in this patient was a challenge we considered that the benefits exceeded the risks and surpassed limitations of other techniques. The anaesthetic plan was sustained by careful communication with the patient and strict collaboration of the surgical team.

Conclusions CSA allowed a safe and effective management during the intraoperative period and optimized the postoperative recovery.

\section{TEMPORARY MENINGEAL SYMPTOMS AFTER EPIDURAL} BLOOD PATCH

E Oreshnikov*, S Oreshnikova, L Kashaeva. Chuvash State University, Cheboksary, Russian Federation

10.1136/rapm-2021-ESRA.56
Background and Aims Post-puncture headache - an unpleasant rare event. Associated with epidural and spinal anesthesia. With epidural anesthesia, its cause is an unintentional puncture of the inner leaf of the dura mater. With spinal, intrathecal puncture itself.

Methods 20 years of experience in post-puncture headache in a maternity hospital ( 200 observations), associated with epidural analgesia in childbirth.

Results Almost 100\% of observations - unintentional puncture of the inner leaf of the dura mater with an epidural needle. Manifests 6-18 hours after removal of the epidural catheter. In less than $50 \%$ of cases amenable to conservative treatment. Classical triad (bedrest, hydration, tylenol) - temporary unstable relief. Most have an epidural blood patch. In case of refusal, contraindications for filling, strict adherence to bed rest for 10-14 days. A single epidural blood patch with autoblood with a volume of $10-20 \mathrm{ml}$ in almost $100 \%$ permanently relieves a headache. Repeated epidural autologous blood filling is required only in $1-2 \%$ of cases. In $1-2 \%$ of our cases of epidural blood patch, we observed short-term, up to 3-5 days, development of meningeal symptoms, in particular, pronounced occipital rigidity against the background of complete disappearance of positional headache. This occipital stiffness looks quite frightening, but usually practically does not bother patients and regresses spontaneously by 5 days after a blood patch.

Conclusions A possible explanation for the development of meningeal symptoms immediately after an epidural patch is the ingestion of a small amount of autologous blood into the subarachnoid space, followed by irritation of the dura mater.

\section{CONTINUOUS SPINAL ANESTHESIA FOR KIDNEY TRANSPLANTATION IN SEVERE MITRAL REGURGITATION LUPIC PATIENT}

${ }^{1}$ A Almeida Pires*, ${ }^{1} \mathrm{M}$ Jesus Correia, ${ }^{2} \mathrm{~J}$ Sousa. ${ }^{1}$ Department of Anesthesiology, Centro Hospitalar de Lisboa Ocidental EPE, Lisboa, Portugal; ' ${ }^{2}$ Department of Cardiology, Centro Hospitalar de Lisboa Ocidental EPE, Lisboa, Portugal

\subsection{6/rapm-2021-ESRA.57}

Background and Aims Anesthesia for kidney transplant on a patient with severe mitral regurgitation is a true challenge since a judicious hemodynamic management is needed to avoid pulmonary edema and ensure optimized reperfusion of the transplanted organ. A 49-year-old Caucasian female with chronic severe mitral regurgitation was scheduled for urgent kidney transplant due to end-stage renal disease caused by lupus nephritis.

Methods We performed the first described continuous spinal anesthesia in a patient with chronic severe mitral regurgitation undergoing cadaver donor kidney transplant. Echocardiography and seriated blood gas analysis were monitored.

Results Cardiac output monitoring confirmed absence of impaired ventricular function or worsening of mitral regurgitation during the installation of subarachnoid block. We used a total of $10 \mathrm{mg}$ bupivacaine and $10 \mathrm{mg}$ sufentanyl and had no need of vasopressor use during block installation and all the intra-operative period. No sedation was administered.

Conclusions Continuous spinal anesthesia provides potential advantages over other forms of neuraxial anesthesia, since low doses of local anesthetic could be titrated during all the 